\title{
Biography for Benzhong Tang
}

\author{
Wai-Yeung Wong
}

Received: 4 November 2014/ Accepted: 5 November 2014/Published online: 29 November 2014

(C) Springer Science+Business Media New York 2014

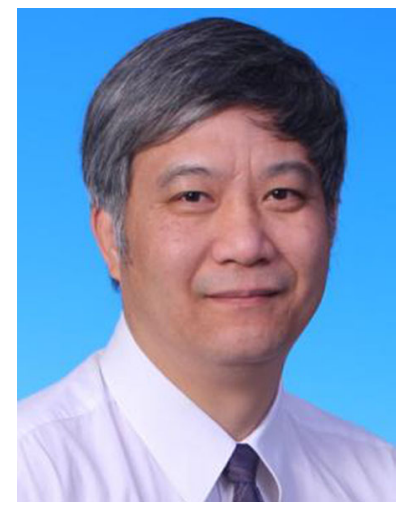

Ben Zhong Tang received his BS and $\mathrm{PhD}$ degrees from South China University of Technology (SCUT) and Kyoto University, respectively. He conducted postdoctoral research at University of Toronto and worked as a senior scientist in Neos Co. Ltd. in 1989-1994. He joined Department of Chemistry at the Hong Kong University of Science \& Technology (HKUST) as an Assistant Professor in 1994 and was promoted to Chair Professor in 2008. He is Chair Professor in the Department of Chemistry and Division of Biomedical Engineering, Stephen K. C. Cheong Professor of Science at HKUST, and also honorary professor at SCUT. He was elected to the Chinese Academy of Sciences in 2009.

$\mathrm{He}$ has published $>600$ papers. His work has been cited over 20,000 times, with an h-index of 80 . He is listed by Thomas Reuters in 2014 as a Highly Cited Researcher in the categories of Chemistry and Materials Science. He has opened a new area of research on aggregation-induced emission (AIE), which was ranked as one of the Top 100 Research Fronts by Thomas Reuters in 2013. He received a State Natural Science Award from Chinese Government in 2007. He is serving as Editor of Advances in Polymer Science (Springer) and Associate Editor of Polymer Chemistry (RSC) and is on the editorial board of a dozen journals. He has made significant contribution to the development of new molecules (particularly those exhibiting AIE features) with novel structures and unique properties with implications for high-tech applications.

Because of his remarkable contributions in the field of polymer science, this special issue will be dedicated to him.

Guest Editors

Wai-Kin Chan

Wai-Yeung Wong

W.-Y. Wong ( $\square)$

Department of Chemistry, Hong Kong Baptist University,

Hong Kong, People's Republic of China

e-mail: rwywong@hkbu.edu.hk 